\title{
Explicit pseudo-transient continuation and the trust-region updating strategy for unconstrained optimization
}

\author{
Xin-long Luo* · Hang Xiao · Jia-hui Lv · Sen \\ Zhang
}

Received: date / Accepted: date

\begin{abstract}
This paper considers an explicit continuation method and the trust-region updating strategy for the unconstrained optimization problem. Moreover, in order to improve its computational efficiency and robustness, the new method uses the switching preconditioning technique. In the well-conditioned phase, the new method uses the L-BFGS method as the preconditioning technique in order to improve its computational efficiency. Otherwise, the new method uses the inverse of the Hessian matrix as the pre-conditioner in order to improve its robustness. Numerical results aslo show that the new method is more robust and faster than the traditional optimization method such as the trust-region method and the line search method. The computational time of the new method is about one percent of that of the trust-region method (the subroutine fminunc.m of the MATLAB2019a environment, it is set by the trustregion method) or one fifth of that the line search method (fminunc.m is set by the
\end{abstract}

\author{
Xin-long Luo \\ Corresponding author. School of Artificial Intelligence, \\ Beijing University of Posts and Telecommunications, P. O. Box 101, \\ Xitucheng Road No. 10, Haidian District, 100876, Beijing China \\ E-mail: luoxinlong@bupt.edu.cn \\ Hang Xiao \\ School of Artificial Intelligence, \\ Beijing University of Posts and Telecommunications, P. O. Box 101, \\ Xitucheng Road No. 10, Haidian District, 100876, Beijing China \\ E-mail: xiaohang0210@bupt.edu.cn \\ Jia-hui Lv \\ School of Artificial Intelligence, \\ Beijing University of Posts and Telecommunications, P. O. Box 101, \\ Xitucheng Road No. 10, Haidian District, 100876, Beijing China \\ E-mail: jhlv@bupt.edu.cn \\ Sen Zhang \\ School of Artificial Intelligence, \\ Beijing University of Posts and Telecommunications, P. O. Box 101, \\ Xitucheng Road No. 10, Haidian District, 100876, Beijing China \\ E-mail: senzhang@bupt.edu.cn
}


quasi-Newton method) for the large-scale problem. Finally, the global convergence analysis of the new method is also given.

Keywords continuation method · trust-region method · line search method · preconditioning technique - generalized gradient flow - unconstrained optimization · quasi-Newton method

Mathematics Subject Classification (2010) $65 \mathrm{~K} 05 \cdot 65 \mathrm{~L} 05 \cdot 65 \mathrm{~L} 20$

\section{Introduction}

In this article, we consider the following unconstrained optimization problem

$$
\min _{x \in \mathfrak{R}^{n}} f(x),
$$

where $f: \Re^{n} \rightarrow \mathfrak{R}$ is a differentiable function. For this problem, there are many efficient methods to solve it such as the line search method and the trust-region method $[12,33,39,44]$. The continuation method $[2,4,17,20,23,24,41]$ is another method other than the traditional optimization methods for the problem (1). The advantage of the continuation method over the line search method or the trust-region method is capable of finding many local optimal points of the non-convex optimization problem by tracking its trajectory, and it is even possible to find the global optimal solution [7, $35,43]$. However, the computational time of the continuation method may be higher than that of the traditional optimization method.

Recently, Luo, Xiao and Lv [28] consider the continuation Newton method with the adaptive time-stepping size based on the trust-region updating strategy for the nonlinear system of equations. According to their numerical experiments, their method is robust and efficient to solve the nonlinear system of equations. In order to improve the computational efficiency and the robustness of their continuation method [28] further for the large-scale optimization problem, we consider the switching preconditioning technique for the ill-conditioned problem. That is to say, in the wellconditioned phase, the new method uses the L-BFGS method as the preconditioning technique in order to improve its computational efficiency. Otherwise, the new method uses the inverse of the Hessian matrix as the pre-conditioner in order to improve its robustness.

The rest of the paper is organized as follows. In section 2, we give a new continuation method with the trust-region updating strategy and the switching preconditioning technique for the unconstrained optimization problem (1). In section 3, we analyze the global convergence of this new method. In section 4, we report some promising numerical results of the new method, in comparison to the traditional trust-region method (fminunc.m is set by the trust-region method) and the line search method (fminunc.m is set by the quasi-Newton method) for some large-scale problems. Finally, we give some discussions and conclusions in section 5 . 


\section{Explicit pseudo-transient continuation}

In this section, we construct an explicit continuation method with the adaptive timestepping scheme based on the trust-region updating strategy [44] for the unconstrained optimization problem (1). Firstly, we construct a generalized gradient flow for the stationary point of the unconstrained optimization (1). Then, we construct an explicit continuation method with an adaptive time-stepping scheme for this special ordinary differential equations (ODEs). In order to improve the robustness of the new method, we also consider a switching preconditioning technique between the L-BFGS method and the inverse of the Hessian matrix.

\subsection{The generalized gradient flow}

For the unconstrained optimization problem (1), we consider the damped Newton method [39] as follows:

$$
x_{k+1}=x_{k}-\alpha_{k} B\left(x_{k}\right)^{-1} g(x),
$$

where $B(x)=\nabla^{2} f(x)$ and $g(x)=\nabla f(x)$. If we regard $x_{k}=x\left(t_{k}\right), x_{k+1}=x\left(t_{k}+\alpha_{k}\right)$ and let $\alpha_{k} \rightarrow 0$, we obtain the continuous Newton flow [5, 14,40] as follows:

$$
\frac{d x}{d t}=-B(x)^{-1} g(x), x(0)=x_{0} .
$$

Actually, if we apply an iteration with the explicit Euler method [38] for the continuous Newton flow (3), we obtain the damped Newton method (2). Since $B(x)$ may be singular, we reformulate the continuous Newton flow (3) as the following general formula:

$$
B(x) \frac{d x}{d t}+g(x)=0, x(0)=x_{0} .
$$

For the continuous Newton flow (4), we have the following property 1.

Property 1 (Branin [5] and Tanabe [40]) Assume that $x(t)$ is the solution of the continuous Newton flow (4), then $r(x(t))=\|g(x(t))\|^{2}$ converges to zero when $t \rightarrow \infty$. That is to say, for every limit point $x^{*}$ of $x(t)$, it is also a stationary point of the Newton flow (4) and every element $g_{i}(x(t))$ of $g(x(t))$ has the same convergence rate $e^{-t}$. Furthermore, $x(t)$ can not converge to the stationary point $x^{*}$ of the continuous Newton flow (4) on the finite interval when the initial point $x_{0}$ is not its stationary point.

Proof. Assume that $x(t)$ is the solution of the continuous Newton flow (4), then we have

$$
\frac{d}{d t}\left(e^{t} g(x)\right)=e^{t} B(x) \frac{d x(t)}{d t}+e^{t} g(x)=0
$$


Consequently, we obtain

$$
g(x(t))=g\left(x_{0}\right) e^{-t}
$$

From equation (5), it is not difficult to know that every element $g_{i}(x(t))$ of $g(x(t))$ converges to zero with the linear convergence rate $e^{-t}$ when $t \rightarrow \infty$. Thus, if the solution $x(t)$ of the continuous Newton flow (4) belongs to a compact set, it has a limit point $x^{*}$ when $t \rightarrow \infty$, and this limit point $x^{*}$ is also its stationary point.

If we assume that the solution $x(t)$ of the continuous Newton flow (4) converges to its stationary point $x^{*}$ on the finite interval $(0, T]$, from equation (5), we have

$$
g\left(x^{*}\right)=g\left(x_{0}\right) e^{-T}
$$

Since $x^{*}$ is a stationary point of the continuous Newton flow (4), we have $g\left(x^{*}\right)=0$. By substituting it into equation (6), we obtain

$$
g\left(x_{0}\right)=0
$$

Thus, it contradicts the assumption that $x_{0}$ is not a stationary point of the continuous Newton flow (4). Therefore, the solution $x(t)$ of the continuous Newton flow (4) can not converge to its stable point $x^{*}$ on the finite interval.

We can also regard the continuous Newton flow as the generalized gradient flow (p. 361, [20]):

$$
\frac{d x}{d t}=-H(x) g(x), x(0)=x_{0}
$$

where $H(x)$ equals the inverse $B(x)^{-1}$ of the Hessian matrix or its quasi-Newton approximation. $H(x)$ can be regarded as a pre-conditioner of $g(x)$ to mitigate the stiffness of the ODEs (7). Consequently, we can adopt the explicit method to compute the trajectory of the ODEs (7) efficiently [28].

Remark 1 If we assume that $x(t)$ is the solution of the ODEs (7) and $H(x)$ is a symmetric positive definite matrix, we obtain

$$
\frac{d f(x)}{d t}=g(x)^{T} \frac{d x}{d t}=-g(x)^{T} H(x) g(x) \leq 0
$$

That is to say, $f(x)$ is monotonically decreasing along the solution curve $x(t)$ of the dynamical system (7). Furthermore, the solution $x(t)$ converges to $x^{*}$ when $f(x)$ is lower bounded and $t$ tends to infinity $[20,24,35,41]$, where $x^{*}$ is the stationary point of the generalized gradient flow (7). Thus, we can follow the trajectory $x(t)$ of the ODEs (7) to obtain its stationary point $x^{*}$, which is also one stationary point of the original optimization problem (1). 
2.2 The explicit continuation method

The solution curve $x(t)$ of the OEDs (7) can not be efficiently followed on an infinite interval by the traditional ODE method $[3,8,21,38]$, so we need to construct the particular method for this problem (7). We apply the first-order implicit Euler method $[3,38]$ to the ODEs (7), then we obtain

$$
x_{k+1}=x_{k}-\Delta t_{k} H\left(x_{k+1}\right) g\left(x_{k+1}\right),
$$

where $\Delta t_{k}$ is the time-stepping size.

Since the system of equations (8) is a nonlinear system which can not be directly solved, we seek for its explicit approximation formula. We denote $s_{k}=x_{k+1}-x_{k}$. By using the first-order Taylor expansion, we have the linear approximation $g\left(x_{k}\right)+$ $B\left(x_{k}\right) s_{k}$ of $g\left(x_{k+1}\right)$. By substituting it into equation (8) and using the zero-order approximation $H\left(x_{k}\right)$ of $H\left(x_{k+1}\right)$, we have

$$
s_{k} \approx-\Delta t_{k} H\left(x_{k}\right)\left(g\left(x_{k}\right)+B\left(x_{k}\right) s_{k}\right)=-\Delta t_{k} H\left(x_{k}\right) g\left(x_{k}\right)-\Delta t_{k} H\left(x_{k}\right) B\left(x_{k}\right) s_{k} .
$$

Let $H\left(x_{k}\right)=\left(\nabla^{2} f\left(x_{k}\right)\right)^{-1}$. Then, we have $H\left(x_{k}\right) B\left(x_{k}\right)=I$. By substituting it into equation (9), we obtain the explicit continuation method as follows:

$$
\begin{aligned}
& s_{k}^{N}=-H_{k} g_{k}, s_{k}=\frac{\Delta t_{k}}{1+\Delta t_{k}} s_{k}^{N}, \\
& x_{k+1}=x_{k}+s_{k},
\end{aligned}
$$

where $g_{k}=\nabla f\left(x_{k}\right)$ and $H_{k}=\left(\nabla^{2} f\left(x_{k}\right)\right)^{-1}$ or its quasi-Newton approximation.

Remark 2 The explicit continuation method (10)-(11) equals the damped Newton method if we let $\alpha_{k}=\Delta t_{k} /\left(1+\Delta t_{k}\right)$ in equation (10). However, from the view of the ODE method, they are different. The damped Newton method (2) is derived from the explicit Euler method applied to the generalized gradient flow (7). Its time-stepping size $\alpha_{k}$ is restricted by the numerical stability [38]. That is to say, for the linear test equation $d x / d t=-\lambda x$, its time-stepping size $\alpha_{k}$ is restricted by the stable region $\left|1-\lambda \alpha_{k}\right| \leq 1$. Therefore, the large time-stepping size $\alpha_{k}$ can not be adopted in the steady-state phase.

The explicit continuation method (10)-(11) is derived from the implicit Euler scheme and the linear approximation $g\left(x_{k}\right)+B\left(x_{k}\right) s_{k}$ of $g\left(x_{k+1}\right)$ applied to the generalized gradient flow (7), and its time-stepping size $\Delta t_{k}$ is not restricted by the numerical stability for the linear test equation. Therefore, the large time-stepping size $\Delta t_{k}$ can be adopted in the steady-state phase, and the explicit continuation method (10)(11) mimics the Newton method. Consequently, it has the fast convergence rate near the stationary point $x^{*}$ of the generalized gradient flow (7). The most of all, the new time-stepping size $\alpha_{k}=\Delta t_{k} /\left(\Delta t_{k}+1\right)$ is favourable to adopt the trust-region updating strategy for adaptively adjusting the time-stepping size $\Delta t_{k}$ such that the explicit continuation method (10)-(11) accurately tracks the generalized gradient flow (7) in the transient-state phase and achieves the fast convergence rate in the steady-state phase. 
Remark 3 Luo, Xiao, Lv [28] and Luo, Yao [29] have considered the explicit continuation method for nonlinear equations and the linear programming problem when $H_{k}=\left(g^{\prime}\left(x_{k}\right)\right)^{-1}$, respectively. Here, we consider the quasi-Newton approximation $H_{k}$ of $\left(g^{\prime}\left(x_{k}\right)\right)^{-1}$ in equation (11).

\subsection{The trust-region updating strategy}

Another issue is how to adaptively adjust the time-stepping size $\Delta t_{k}$ at every iteration. We borrow the adjustment method of the trust-region radius from the trust-region method due to its robustness and its fast convergence rate $[12,44]$. When we use the trust-region updating strategy to adaptively adjust time-stepping size $\Delta t_{k}[22,25,26$, 27], we also need to construct a local approximation model of $f(x)$ around $x_{k}$. Here, we adopt the following quadratic function as its approximation model:

$$
q_{k}\left(x_{k}+s\right)=f\left(x_{k}\right)+s^{T} g_{k}+\frac{1}{2} s^{T} B_{k} s,
$$

where $B_{k}=\nabla^{2} f\left(x_{k}\right)$ or its quasi-Newton approximation. In practical computation, we do not store the matrix $B_{k}$. Thus, we use the explicit continuation method (10)-(11) and regard $H_{k}=B_{k}^{-1}$ to simplify the quadratic model $q_{k}\left(x_{k}+s_{k}\right)-q\left(x_{k}\right)$ as follows:

$$
m_{k}\left(s_{k}\right)=g_{k}^{T} s_{k}-\frac{0.5 \Delta t_{k}}{1+\Delta t_{k}} g_{k}^{T} s_{k}=\frac{1+0.5 \Delta t_{k}}{1+\Delta t_{k}} g_{k}^{T} s_{k} \approx q_{k}\left(x_{k}+s_{k}\right)-q_{k}\left(x_{k}\right) .
$$

where $g_{k}=\nabla f\left(x_{k}\right)$. We enlarge or reduce the time-stepping size $\Delta t_{k}$ at every iteration according to the following ratio:

$$
\rho_{k}=\frac{f\left(x_{k}\right)-f\left(x_{k+1}\right)}{m_{k}(0)-m_{k}\left(s_{k}\right)} .
$$

A particular adjustment strategy is given as follows:

$$
\Delta t_{k+1}= \begin{cases}\gamma_{1} \Delta t_{k}, & \text { if } 0 \leq\left|1-\rho_{k}\right| \leq \eta_{1}, \\ \Delta t_{k}, & \text { if } \eta_{1}<\left|1-\rho_{k}\right|<\eta_{2}, \\ \gamma_{2} \Delta t_{k}, & \text { if }\left|1-\rho_{k}\right| \geq \eta_{2},\end{cases}
$$

where the constants are selected as $\eta_{1}=0.25, \gamma_{1}=2, \eta_{2}=0.75, \gamma_{2}=0.5$ according to numerical experiments. When $\rho_{k} \geq \eta_{a}$, we accept the trial step $s_{k}$ and let $x_{k+1}=$ $x_{k}+s_{k}$, where $\eta_{a}$ is a small positive number such as $\eta_{a}=1.0 \times 10^{-6}$. Otherwise, we discard it and let $x_{k+1}=x_{k}$.

Remark 4 This new time-stepping size selection based on the trust-region strategy has some advantages compared to the traditional line search strategy. If we use the line search strategy and the damped Newton method (2) to track the trajectory $x(t)$ of the continuous Newton flow (4), in order to achieve the fast convergence rate in the steady-state phase, the time-stepping size $\alpha_{k}$ of the damped Newton method is 
tried from 1 and reduced by the half with many times at every iteration. Since the linear model $f\left(x_{k}\right)+g_{k}^{T} s_{k}$ may not approximate $f\left(x_{k}+s_{k}\right)$ well in the transient-state phase, the time-stepping size $\alpha_{k}$ will be small. Consequently, the line search strategy consumes the unnecessary trial steps in the transient-state phase. However, the selection of the time-stepping size $\Delta t_{k}$ based on the trust-region strategy (14)-(15) can overcome this shortcoming.

\subsection{The switching preconditioning technique}

For the large-scale problem, the numerical evaluation of the Hessian matrix $\nabla^{2} f\left(x_{k}\right)$ consumes much time. In order to overcome this shortcoming, we use the limitedmemory BFGS quasi-Newton formula (see [6,15,18,32,36] or pp. 222-230, [33]) to approximate $\left(\nabla^{2} f\left(x_{k}\right)\right)^{-1}$.

Recently, Ullah, Sabi and Shah [42] give an efficient L-BFGS updating formula for the system of monotone nonlinear equations. In order to avoid the ill-conditioning of $B_{k}$, they considered the revised BFGS updating formula [42] as follows:

$$
B_{k+1}=\lambda_{k}\left(I-\frac{s_{k} s_{k}^{T}}{s_{k}^{T} s_{k}}\right)+\sigma_{k} \frac{y_{k} y_{k}^{T}}{y_{k}^{T} s_{k}},
$$

where $y_{k}=g_{k+1}-g_{k}, s_{k}=x_{k+1}-x_{k}$ and $\lambda_{k}, \sigma_{k}$ are two undetermined parameters. Then, they solved the minimizer of the measurement function $\varphi$ [9] on the variables $\lambda_{k}$ and $\sigma_{k}$, where $\varphi\left(B_{k+1}\right)$ is defined by

$$
\begin{aligned}
& \varphi\left(\lambda_{k}, \sigma_{k}\right)=\Psi\left(B_{k+1}\right)=\operatorname{trace}\left(B_{k+1}\right)-\ln \left(\operatorname{det}\left(B_{k+1}\right)\right) \\
& \quad=(n-1) \lambda_{k}+\sigma_{k} \frac{\left\|y_{k}\right\|^{2}}{y_{k}^{T} s_{k}}-\ln \left(\lambda_{k}^{n-1} \sigma_{k} \frac{y_{k}^{T} s_{k}}{\left\|s_{k}\right\|^{2}}\right) \\
& =(n-1)\left(\lambda_{k}-\ln \left(\lambda_{k}\right)\right)+\sigma_{k} \frac{\left\|y_{k}\right\|^{2}}{y_{k}^{T} s_{k}}-\ln \left(\sigma_{k}\right)-\ln \left(y_{k}^{T} s_{k}\right)+\ln \left(\left\|s_{k}\right\|^{2}\right) .
\end{aligned}
$$

Consequently, by solving $\min \varphi\left(\lambda_{k}, \sigma_{k}\right)$, they obtained the optimal parameters $\lambda_{k}=$ 1 and $\sigma_{k}=y_{k}^{T} s_{k} /\left\|y_{k}\right\|^{2}$. By substituting them into equation (16), they obtained the revised L-BFGS updating formula:

$$
B_{k+1}=I-\frac{s_{k} s_{k}^{T}}{s_{k}^{T} s_{k}}+\frac{y_{k} y_{k}^{T}}{\left\|y_{k}\right\|^{2}}
$$

By using the Sherman-Morrison-Woodburg formula (P. 17, [39]), from equation (18), we obtain the inverse of $B_{k+1}$ :

$$
H_{k+1}=B_{k+1}^{-1}=I-\frac{y_{k} s_{k}^{T}+s_{k} y_{k}^{T}}{y_{k}^{T} s_{k}}+2 \frac{y_{k}^{T} y_{k}}{\left(y_{k}^{T} s_{k}\right)^{2}} s_{k} s_{k}^{T},
$$


The initial matrix $H_{0}$ can be simply selected by the inverse $\left(\nabla^{2} f\left(x_{0}\right)\right)^{-1}$ of the Hessian matrix. From equation (19), it is not difficult to verify

$$
H_{k+1} y_{k}=\frac{y_{k}^{T} y_{k}}{y_{k}^{T} s_{k}} s_{k} .
$$

That is to say, $H_{k+1}$ satisfies the scaling quasi-Newton property.

The L-BFGS updating formula (19) has some nice properties such as the symmetric positive definite property and the positive lower bound of its eigenvalues.

Lemma 1 Matrix $H_{k+1}$ defined by equation (19) is symmetric positive definite and its eigenvalues are greater than $1 / 2$.

Proof. (i) For any nonzero vector $z \in \mathfrak{R}^{n}$, from equation (19), we have

$$
\begin{aligned}
& z^{T} H_{k+1} z=\|z\|^{2}-2\left(z^{T} y_{k}\right)\left(z^{T} s_{k}\right) / y_{k}^{T} s_{k}+2\left(z^{T} s_{k}\right)^{2}\left\|y_{k}\right\|^{2} /\left(y_{k}^{T} s_{k}\right)^{2} \\
& =\left(\|z\|-\left|z^{T} s_{k} / y_{k}^{T} s_{k}\right|\left\|y_{k}\right\|\right)^{2}+2\|z\|\left|z^{T} s_{k} / y_{k}^{T} s_{k}\right|\left\|y_{k}\right\| \\
& \quad-2\left(z^{T} y_{k}\right)\left(z^{T} s_{k}\right) / y_{k}^{T} s_{k}+\left\|y_{k}\right\|^{2}\left(z^{T} s_{k} / y_{k}^{T} s_{k}\right)^{2} \geq 0 .
\end{aligned}
$$

In the last inequality of equation (20), we use the Cauchy-Schwartz inequality $\left\|z^{T} y\right\| \leq$ $\|z\|\left\|y_{k}\right\|$ and its equality holds if only if $z=t y_{k}$. When $z=t y_{k}$, from equation (20), we have $z^{T} H_{k+1} z=t^{2}\left\|y_{k}\right\|^{2}=\|z\|^{2}>0$. When $z^{T} s_{k}=0$, from equation (20), we also have $z^{T} H_{k+1} z=\|z\|^{2}>0$. Therefore, we conclude that $H_{k+1}$ is a symmetric positive definite matrix.

(ii) It is not difficult to know that it exists at least $n-2$ linearly independent vectors $z_{1}, z_{2}, \ldots, z_{n-2}$ such that $z_{i}^{T} s_{k}=0, z_{i}^{T} y_{k}=0(i=1,2, \ldots,(n-2))$ hold. That is to say, matrix $H_{k+1}$ defined by equation (19) has at least $(n-2)$ linearly independent eigenvectors whose corresponding eigenvalues are 1 . We denote the other two eigenvalues of $H_{k+1}$ as $\mu_{i}^{k+1}(i=1,2)$ and their corresponding eigenvalues as $p_{1}$ and $p_{2}$, respectively. Then, from equation (19), we know that the eigenvectors $p_{i}(i=1,2)$ can be represented as $p_{i}=y_{k}+\beta_{i} s_{k}$ when $\mu_{i}^{k+1} \neq 1(i=1,2)$. From equation (19) and $H_{k+1} p_{i}=\mu_{i}^{k+1} p_{i}(i=1,2)$, we have

$$
-\left(\mu_{i}^{k+1}+\beta_{i} \frac{s_{k}^{T} s_{k}}{s_{k}^{T} y_{k}}\right) y_{k}+\left(\frac{y_{k}^{T} y_{k}}{y_{k}^{T} s_{k}}+2 \beta_{i} \frac{\left(y_{k}^{T} y_{k}\right)\left(s_{k}^{T} s_{k}\right)}{\left(y_{k}^{T} s_{k}\right)^{2}}-\mu_{i}^{k+1} \beta_{i}\right) s_{k}=0 .
$$

When $y_{k}=t s_{k}$, from equation (19), we have $H_{k+1}=I$. In this case, we conclude that the eigenvalues of $H_{k+1}$ are greater than $1 / 2$. When vectors $y_{k}$ and $s_{k}$ are linearly independent, from equation (21), we have

$$
\begin{aligned}
& \mu_{i}^{k+1}+\beta_{i} s_{k}^{T} s_{k} / s_{k}^{T} y_{k}=0, \\
& y_{k}^{T} y_{k} / y_{k}^{T} s_{k}+2 \beta_{i}\left(y_{k}^{T} y_{k}\right)\left(s_{k}^{T} s_{k}\right) /\left(y_{k}^{T} s_{k}\right)^{2}-\mu_{i}^{k+1} \beta_{i}=0, i=1,2 .
\end{aligned}
$$

That is to say, $\mu_{i}^{k+1}(i=1: 2)$ are the two solutions of the following equation:

$$
\mu^{2}-2 \mu\left(y_{k}^{T} y_{k}\right)\left(s_{k}^{T} s_{k}\right) /\left(s_{k}^{T} y_{k}\right)^{2}+\left(y_{k}^{T} y_{k}\right)\left(s_{k}^{T} s_{k}\right) /\left(s_{k}^{T} y_{k}\right)^{2}=0 .
$$


Consequently, from equation (22), we obtain

$$
\mu_{1}^{k+1}+\mu_{2}^{k+1}=2\left(y_{k}^{T} y_{k}\right)\left(s_{k}^{T} s_{k}\right) /\left(s_{k}^{T} y_{k}\right)^{2}, \mu_{1}^{k+1} \mu_{2}^{k+1}=\left(y_{k}^{T} y_{k}\right)\left(s_{k}^{T} s_{k}\right) /\left(s_{k}^{T} y_{k}\right)^{2} .
$$

From equation (23), it is not difficult to obtain

$$
1 / \mu_{1}^{k+1}+1 / \mu_{2}^{k+1}=2, \mu_{i}^{k+1}>0, i=1,2
$$

Therefore, from equation (24), we conclude that $\mu_{i}^{k+1}>\frac{1}{2}(i=1,2)$. Consequently, the eigenvalues of $H_{k+1}$ are greater than $1 / 2$.

According to our numerical experiments, the quasi-Newton updating method (19) works well for the most unconstrained optimization problems. However, for the very ill-conditioned problem, the quasi-Newton method (19) will fail to accurately obtain its minimizer $x^{*}$. In order to improve the robustness of the method, we use the inverse $\left(\nabla^{2} f\left(x_{k}\right)\right)^{-1}$ of the Hessian matrix as the pre-conditioner of the gradient $g\left(x_{k}\right)$ in the ill-conditioned phase. Furthermore, we identify the ill-conditioned phase by the gap between $f\left(x_{k}+s_{k}\right)$ and its approximation $f\left(x_{k}\right)+g\left(x_{k}\right)^{T} s_{k}+\frac{1}{2} s_{k}^{T} B_{k} s_{k}$ when $s_{k}$ is small. That is to say, we regard the problem as an ill-conditioned phase when the number of $k$ that satisfies $\left|1-\rho_{k}\right| \geq \eta_{2}$ is greater than the threshold. Therefore, we give the following switching preconditioning technique as follows:

$$
H_{k+1}=\left\{\begin{array}{l}
I-\frac{y_{k} s_{k}^{T}+s_{k} y_{k}^{T}}{y_{k}^{T} s_{k}}+2 \frac{y_{k}^{T} y_{k}}{\left(y_{k}^{T} s_{k}\right)^{2}} s_{k} s_{k}^{T}, \text { if } K_{b a d} \leq 5 \text { and }\left|s_{k}^{T} y_{k}\right|>\theta\left\|s_{k}\right\|^{2} \\
\left(\nabla^{2} f\left(x_{k+1}\right)\right)^{-1}, \text { otherwise, }
\end{array}\right.
$$

where $K_{b a d}$ represents the number of $k$ such that $\left|1-\rho_{k}\right| \geq \eta_{2}$ holds, the ratio $\rho_{k}$ is defined by equation (14), and $\theta$ is a small positive constant such as $\theta=10^{-6}$.

For a real-world problem, the analytical Hessian $\nabla^{2} f(x)$ can not be offered. Thus, in practice, we replace the Hessian matrix $\nabla^{2} f\left(x_{k+1}\right)$ with its difference approximation as follows:

$$
\nabla^{2} f\left(x_{k+1}\right) \approx\left[\frac{g\left(x_{k+1}+\varepsilon e_{1}\right)-g\left(x_{k+1}\right)}{\varepsilon}, \ldots, \frac{g\left(x_{k+1}+\varepsilon e_{n}\right)-g\left(x_{k+1}\right)}{\varepsilon}\right],
$$

where $e_{i}$ represents the unit vector whose elements equal zeros except for the $i$-th element equals 1 , and the parameter $\varepsilon$ can be selected as $10^{-6}$ according to our numerical experiments.

According to the above discussions, we give the detailed implementation of the explicit continuation method with the trust-region updating strategy for the unconstrained optimization problem (1) in Algorithm 1.

\section{Algorithm analysis}

In this section, we analyze the global convergence of the explicit continuation method (10)-(11) with the trust-region updating strategy and the switching preconditioning technique (25) for the unconstrained optimization problem (i.e. Algorithm 1). Firstly, 


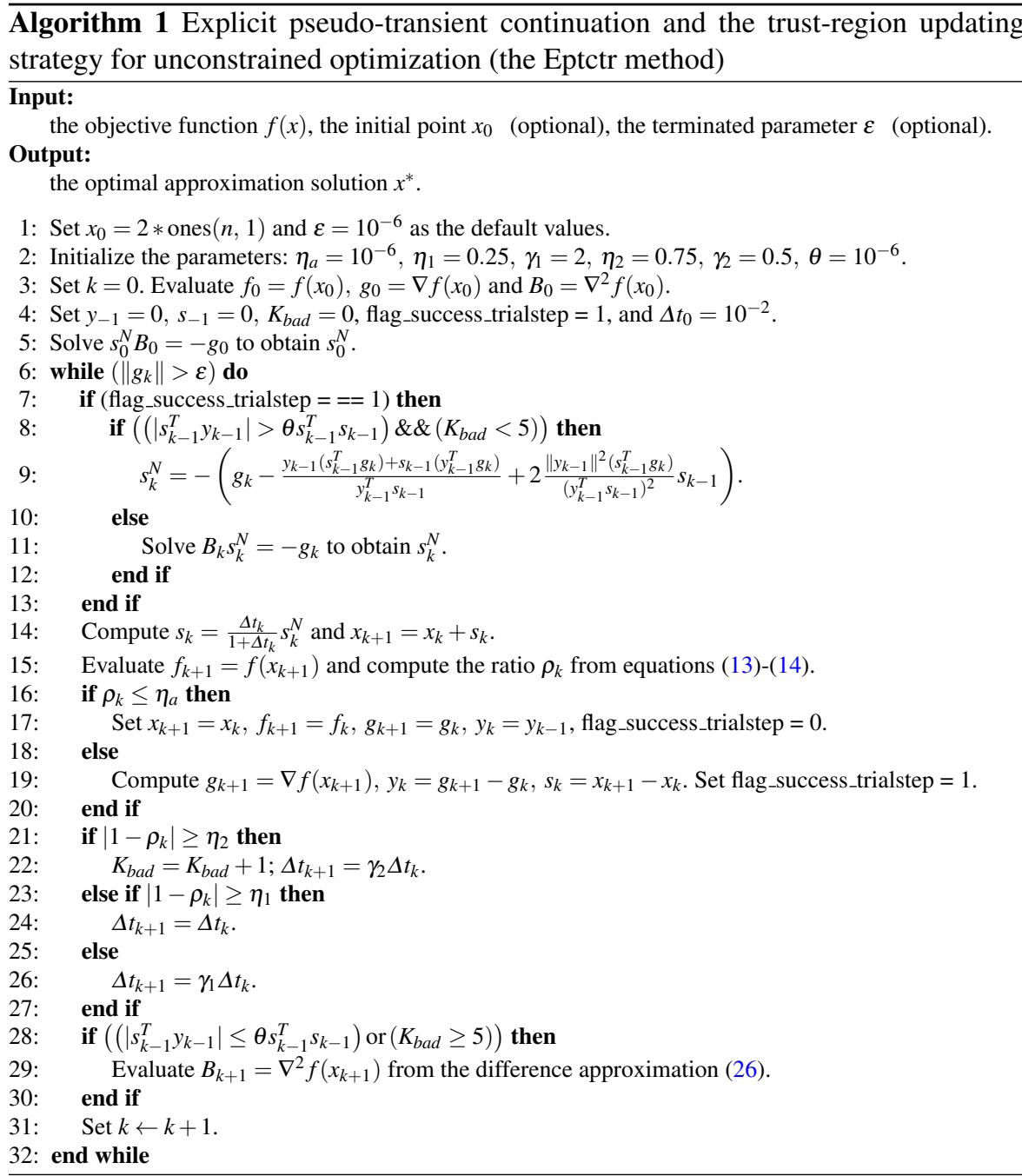

we give a lower-bounded estimation of $m_{k}(0)-m_{k}\left(s_{k}\right)(k=1,2, \ldots)$. This result is similar to that of the trust-region method for the unconstrained optimization problem [34]. We denote the level set $S_{f}$ as

$$
S_{f}=\left\{x: f(x) \leq f\left(x_{0}\right)\right\} .
$$

Lemma 2 Assume that there exist two positive constants $m$ and $M$ such that

$$
M\|z\|^{2} \geq z^{T} \nabla^{2} f(x) z \geq m\|z\|^{2}>0, \forall x \in S_{f}
$$

holds for all $z \in \mathfrak{R}^{n}$. Furthermore, we assume that the quadratic model $q_{k}(x)$ is defined by equation (13) and $s_{k}$ is computed by the explicit continuation method (10)- 
(11) and the switching preconditioning formula (25). Then, we have

$$
m_{k}(0)-m_{k}\left(s_{k}\right) \geq \frac{c_{m} \Delta t_{k}}{2\left(1+\Delta t_{k}\right)}\left\|g_{k}\right\|^{2}
$$

where $c_{m}$ is a positive constant.

Proof. When $H_{k}$ is updated by the L-BFGS formula (19), from Lemma 1, we know that $H_{k}$ is symmetric positive definite and its eigenvalues are greater than $1 / 2$. When $H_{k}=\left(\nabla^{2} f\left(x_{k}\right)\right)^{-1}$, from the assumption (28) of $\nabla^{2} f\left(x_{k}\right)$, we know that $H_{k}$ is symmetric positive definite and its eigenvalues are greater than $1 / M$. By combining these two cases, we know that the eigenvalues of $H_{k}$ are greater than $c_{m}=\min \{1 / 2,1 / M\}$.

By using the eigenvalue decomposition of $H_{k}$, from the explicit continuation method (10)-(11) and the quadratic model (13), we have

$$
m_{k}(0)-m_{k}\left(s_{k}\right) \geq-\frac{1}{2} g_{k}^{T} s_{k}=\frac{\Delta t_{k}}{2\left(1+\Delta t_{k}\right)} g_{k}^{T} H_{k} g_{k}=\frac{c_{m} \Delta t_{k}}{2\left(1+\Delta t_{k}\right)}\left\|g_{k}\right\|^{2} .
$$

In the first inequality in equation (30), we use the property $\left(1+0.5 \Delta t_{k}\right) /\left(1+\Delta t_{k}\right) \geq$ 0.5 when $\Delta t_{k} \geq 0$. Consequently, we prove the result (29).

In order to prove that $p_{g_{k}}$ converges to zero when $k$ tends to infinity, we need to estimate the lower bound of time-stepping sizes $\Delta t_{k}(k=1,2, \ldots)$.

Lemma 3 Assume that $f: \Re^{n} \rightarrow \mathfrak{R}$ is twice continuously differentiable and its gradient $g(x)$ satisfies the following Lipschitz continuity:

$$
\|g(x)-g(y)\| \leq L_{C}\|x-y\|, \forall x, y \in S_{f},
$$

where $L_{C}$ is the Lipschitz constant. The Hessian matrix $\nabla^{2} f(x)$ satisfies the strong convexity (28). We suppose that the sequence $\left\{x_{k}\right\}$ is generated by Algorithm 1. Then, there exists a positive constant $\delta_{\Delta t}$ such that

$$
\Delta t_{k} \geq \gamma_{2} \delta_{\Delta t}
$$

holds for all $k=1,2, \ldots$, where $\Delta t_{k}$ is adaptively adjusted by the formulas (13)-(15).

Proof. When $H_{k}$ is updated by the L-BFGS formula (19), from Lemma 1, we know that the eigenvalues of $H_{k}$ is greater than $1 / 2$ and it has at least $n-2$ eigenvalues of 1 . When $\left|s_{k-1}^{T} y_{k-1}\right| \geq \theta\left\|s_{k-1}\right\|^{2}$, we denote the other two eigenvalues of $H_{k}$ as $\mu_{1}^{k}$ and $\mu_{2}^{k}$. By substituting it into equation (23), we obtain

$$
\mu_{1}^{k} \mu_{2}^{k}=\frac{\left\|y_{k-1}\right\|^{2}\left\|s_{k-1}\right\|^{2}}{\left(s_{k-1}^{T} y_{k-1}\right)^{2}} \leq \frac{\left\|y_{k-1}\right\|^{2}\left\|s_{k-1}\right\|^{2}}{\theta^{2}\left\|s_{k-1}\right\|^{4}}=\frac{1}{\theta^{2}} \frac{\left\|y_{k-1}\right\|^{2}}{\left\|s_{k-1}\right\|^{2}} .
$$

From Lemma 2 and Algorithm 1, we know $f\left(x_{k}\right) \leq f\left(x_{0}\right)(k=1,2, \ldots)$. From the Lipschitz continuity $(31)$ of $g(\cdot)$, we have

$$
\left\|y_{k-1}\right\|=\left\|g\left(x_{k}\right)-g\left(x_{k-1}\right)\right\| \leq L_{C}\left\|x_{k}-x_{k-1}\right\|=L_{C}\left\|s_{k-1}\right\| .
$$


By substituting it into equation (33) and using $\mu_{i}^{k}>\frac{1}{2}(i=1,2)$, we obtain

$$
\frac{1}{2}<\mu_{i}^{k}<\frac{2 L_{C}^{2}}{\theta^{2}}, i=1,2 .
$$

That is to say, the eigenvalues of $H_{k}$ are less than or equal to $\max \left\{1,2 L_{C}^{2} / \theta^{2}\right\}$.

When $H_{k}=\left(\nabla^{2} f\left(x_{k}\right)\right)^{-1}$, from the assumption (28) of $\nabla^{2} f\left(x_{k}\right)$, we know that $H_{k}$ is symmetric positive definite and its eigenvalues are less than or equal to $1 / \mathrm{m}$.

By combining these two cases, we obtain that the eigenvalues of $H_{k}$ are less than or equal to $M_{H}$ when $H_{k}$ is updated by the preconditioning formula (25), where $M_{H}=$ $\max \left\{1,2 L_{C}^{2} / \theta^{2}, 1 / m\right\}$. By using the property of the matrix norm, we have

$$
\left\|H_{k} g_{k}\right\| \leq M_{H}\left\|g_{k}\right\| .
$$

From the first-order Taylor expansion, we have

$$
f\left(x_{k}+s_{k}\right)=f\left(x_{k}\right)+\int_{0}^{1} s_{k}^{T} g\left(x_{k}+t s_{k}\right) d t .
$$

Thus, from equations (13)-(14), (29), (37) and the Lipschitz continuity (31) of $g(\cdot)$, we have

$$
\begin{gathered}
\left|\rho_{k}-1\right|=\left|\frac{\left(f\left(x_{k}\right)-f\left(x_{k}+s_{k}\right)\right)-\left(m_{k}(0)-m_{k}\left(s_{k}\right)\right)}{m_{k}(0)-m_{k}\left(s_{k}\right)}\right| \\
\leq \frac{\left|\int_{0}^{1} s_{k}^{T}\left(g\left(x_{k}+t s_{k}\right)-g\left(x_{k}\right)\right) d t\right|}{m_{k}(0)-m_{k}\left(s_{k}\right)}+\frac{0.5 \Delta t_{k}}{1+0.5 \Delta t_{k}} \\
\leq \frac{L_{C}\left(1+\Delta t_{k}\right)}{c_{m} \Delta t_{k}} \frac{\left\|s_{k}\right\|^{2}}{\left\|g_{k}\right\|^{2}}+\frac{0.5 \Delta t_{k}}{1+0.5 \Delta t_{k}} .
\end{gathered}
$$

By substituting equation (10) and equation (36) into equation (38), we have

$$
\begin{aligned}
& \left|\rho_{k}-1\right| \leq \frac{L_{C} \Delta t_{k}}{c_{m}\left(1+\Delta t_{k}\right)} \frac{\left\|H_{k} g_{k}\right\|^{2}}{\left\|g_{k}\right\|^{2}}+\frac{0.5 \Delta t_{k}}{1+0.5 \Delta t_{k}} \\
& \leq \frac{L_{C} \Delta t_{k}}{c_{m}\left(1+\Delta t_{k}\right)} \frac{M_{H}^{2}\left\|g_{k}\right\|^{2}}{\left\|g_{k}\right\|^{2}}+\frac{0.5 \Delta t_{k}}{1+0.5 \Delta t_{k}} \leq \frac{\left(L_{C} M_{H}^{2}+0.5 c_{m}\right) \Delta t_{k}}{c_{m}\left(1+0.5 \Delta t_{k}\right)} .
\end{aligned}
$$

We denote

$$
\delta_{\Delta t} \triangleq \frac{c_{m} \eta_{1}}{L_{C} M_{H}^{2}+0.5 c_{m}} .
$$

Then, from equation (39)-(40), when $\Delta t_{k} \leq \delta_{\Delta t}$, it is not difficult to verify

$$
\left|\rho_{k}-1\right| \leq \frac{L_{C} M_{H}^{2}+0.5 c_{m}}{c_{m}} \Delta t_{k} \leq \eta_{1} .
$$


We assume that $K$ is the first index such that $\Delta t_{K} \leq \delta_{\Delta t}$ where $\delta_{\Delta t}$ is defined by equation (40). Then, from equations (40)-(41), we know that $\left|\rho_{K}-1\right| \leq \eta_{1}$. According to the time-stepping adjustment formula (15), $x_{K}+s_{K}$ will be accepted and the time-stepping size $\Delta t_{K+1}$ will be enlarged. Consequently, the time-stepping size $\Delta t_{k}$ holds $\Delta t_{k} \geq \gamma_{2} \delta_{\Delta t}$ for all $k=1,2, \ldots$.

By using the results of Lemma 2 and Lemma 3, we prove the global convergence of Algorithm 1 for the unconstrained optimization problem (1) as follows.

Theorem 1 Assume that $f: \mathfrak{R}^{n} \rightarrow \mathfrak{R}$ is twice continuously differentiable and its gradient $\nabla f(\cdot)$ satisfies the Lipschitz continuity (31). The Hessian matrix $\nabla^{2} f(x)$ satisfies the strong convexity (28). Furthermore, we suppose that $f(x)$ is lower bounded when $x \in S_{f}$, where the level set $S_{f}$ is defined by equation (27). The sequence $\left\{x_{k}\right\}$ is generated by Algorithm 1. Then, we have

$$
\liminf _{k \rightarrow \infty}\left\|g_{k}\right\|=0 .
$$

Proof. According to Lemma 3 and Algorithm 1, we know that there exists an infinite subsequence $\left\{x_{k_{i}}\right\}$ such that the trial steps $s_{k_{i}}$ are accepted, i.e., $\rho_{k_{i}} \geq \eta_{a}, i=$ $1,2, \ldots$ Otherwise, all steps are rejected after a given iteration index, then the timestepping size will keep decreasing, which contradicts (32). Therefore, from equations (14) and (29), we have

$$
\begin{aligned}
& f\left(x_{0}\right)-\lim _{k \rightarrow \infty} f\left(x_{k}\right)=\sum_{k=0}^{\infty}\left(f\left(x_{k}\right)-f\left(x_{k+1}\right)\right) \\
& \geq \eta_{a} \sum_{i=0}^{\infty}\left(m_{k_{i}}(0)-m_{k_{i}}\left(s_{k_{i}}\right)\right) \geq \eta_{a} \sum_{i=0}^{\infty} \frac{c_{m} \Delta t_{k_{i}}}{2\left(\Delta t_{k_{i}}+1\right)}\left\|g_{k_{i}}\right\| .
\end{aligned}
$$

From the result (32) of Lemma 3, we know that $\Delta t_{k} \geq \gamma_{2} \delta_{\Delta t}(k=1,2, \ldots)$. By substituting it into equation (43), we have

$$
f\left(x_{0}\right)-\lim _{k \rightarrow \infty} f\left(x_{k}\right) \geq \eta_{a} \sum_{i=0}^{\infty} \frac{\gamma_{2} c_{m} \delta_{\Delta t}}{2\left(\gamma_{2} \delta_{\Delta t}+1\right)}\left\|g_{k_{i}}\right\|
$$

Since $f(x)$ is lower bounded when $x \in S_{f}$ and the sequence $\left\{f\left(x_{k}\right)\right\}$ is monotonically decreasing, we have $\lim _{k \rightarrow \infty} f\left(x_{k}\right)=f^{*}$. By substituting it into equation (44), we obtain the result (42).

\section{Numerical experiments}

In this section, some numerical experiments are performed to test the performance of Algorithm 1 (the Eptctr method). The codes are executed by a HP notebook with the Intel quad-core CPU and 8GB RAM. We compare Eptctr with the trust-region method and the line searh method (the built-in subroutine fminunc.m of the MATLAB2019a environment) $[6,10,11,13,16,30]$ for 47 unconstrained optimization problems which can be found in $[1,31,37]$. The trust-region method and the line search method are 
two classical methods for solving unconstrained optimization problems and these two methods have been widely used until today. Therefore, we select these two typical methods as the basis for comparison. The termination conditions of the three compared methods are all set by

$$
\left\|\nabla f\left(x_{k}\right)\right\|_{\infty} \leq 1.0 \times 10^{-6} .
$$

The initial points are set to $x_{0}=2 \times$ ones $(n, 1)$ for all test problems.

The numerical results are arranged in Table 1, Table 2 and Figure 1. From Tables 1-2, we find that Eptctr works well for those 47 test problems. However, the trust-region method (fminunc.m is set by the trust-region method) and the line search method (fminunc.m is set by the quasi-Newton method) fail to solve 6 problems and 13 problems, respectively. Thus, Eptctr is more robust than the traditional optimization method such as the trust-region method and the line search method.

Moreover, from Table 1, we find that the computational time of Eptctr is about one percent of that of the trust-region method (fminunc.m is set by the trust-region method) or one fifth of that the line search method (fminunc.m is set by the quasiNewton method). One of the reasons is that the generalized gradient flow is non-stiff in the transient-state phase and Eptctr uses the L-BFGS method 19 as the preconditioning technique to follow their trajectories well for the most problems. Consequently, Eptctr only involves three pairs of the inner product of two vectors to obtain the trial step $s_{k}$ at every iteration of the transient-state phase.

However, the trust-region method needs at least to solve a linear system of equations and involves about $1 / 3 n^{3}$ flops (p. 169, [19]) at every iteration. Actually, in order to obtain the search step $s_{k}$, the trust-region method needs to solve a trustregion subproblem, which requires to solve a nonlinear system of equations at every iteration. The line search method involves one matrix-vector product to obtain the search direction $d_{k}$ and it involves about $n^{2}$ flops at every iteration. The other reason is that the step size $\alpha_{k}$ of the line search method is tried from 1 and reduced by the half with many times at every iteration. Since the linear model $f\left(x_{k}\right)+g_{k}^{T} s_{k}$ may not approximate $f\left(x_{k}+s_{k}\right)$ well in the transient-state phase, the step size $\alpha_{k}$ will be small. Consequently, the line search strategy consumes the unnecessary trial steps in the transient-state phase.

\section{Conclusions}

For the unconstrained optimization problem, we consider an explicit continuation method with the trust-region updating strategy and the switching preconditioning technique (Eptctr) to solve it. For the well-conditioned phase, Eptctr uses the LBFGS method (19) as the preconditioning technique. Otherwise, Eptctr uses the inverse $\left(\nabla^{2} f\left(x_{k}\right)\right)^{-1}$ of the Hessian matrix as the pre-conditioner in order to improve its robustness. Consequently, for the well-conditioned phase, Eptctr only involves the $6 n$ flops to obtain its trial step $s_{k}$ at every iteration. 
Table 1: Numerical results of large-scale problems with $n=1000$.

\begin{tabular}{|c|c|c|c|c|c|c|}
\hline \multirow{2}{*}{ Problems } & \multicolumn{2}{|c|}{ Eptctr } & \multicolumn{2}{|c|}{ fminunc.m (trust-region) } & \multicolumn{2}{|c|}{ fminunc.m (quasi-Newton) } \\
\hline & Iter (time $(\mathrm{s})$ ) & $\left\|g\left(x^{i t}\right)\right\|_{\infty}$ & Iter (time (s)) & $\left\|g\left(x^{i t}\right)\right\|_{\infty}$ & Iter (time (s)) & $\left\|g\left(x^{i t}\right)\right\|_{\infty}$ \\
\hline $\begin{array}{l}\text { 1. Trid Function } \\
(\mathrm{n}=1000)\end{array}$ & $28(0.2800)$ & $7.7882 \mathrm{E}-07$ & $\begin{array}{c}21 \text { (147.3268) } \\
\text { (failed) }\end{array}$ & 0.0052 & $\begin{array}{l}89(5.8060) \\
\text { (failed) }\end{array}$ & 185.3336 \\
\hline $\begin{array}{l}\text { 2. Rosenbrock Function } \\
(\mathrm{n}=1000)\end{array}$ & $37(0.6280)$ & $1.8925 \mathrm{E}-07$ & $15(208.36)$ & $6.9663 \mathrm{E}-11$ & $23(1.0736)$ & $3.7971 \mathrm{E}-05$ \\
\hline $\begin{array}{l}\text { 3. Ackley Function } \\
(\mathrm{n}=1000)\end{array}$ & $74(0.1168)$ & $2.1729 \mathrm{E}-06$ & $2(52.7352)$ & $6.9663 \mathrm{E}-11$ & $4(0.3761)$ & 0 \\
\hline $\begin{array}{l}\text { 4. Dixon Price Function } \\
(\mathrm{n}=1000)\end{array}$ & $37(0.6220)$ & $1.8582 \mathrm{E}-07$ & $\begin{array}{c}11 \text { (78.2305) } \\
\text { (failed) }\end{array}$ & $5.1937 \mathrm{E}-04$ & $\begin{array}{c}95(4.5570) \\
\text { (failed) }\end{array}$ & 10.3910 \\
\hline $\begin{array}{l}\text { 5. Levy Function } \\
(\mathrm{n}=1000)\end{array}$ & $38(0.1521)$ & $7.6318 \mathrm{E}-07$ & $6(49.5847)$ & $4.9983 \mathrm{E}-13$ & $11(0.8782)$ & $2.5648 \mathrm{E}-07$ \\
\hline $\begin{array}{l}\text { 6. Molecular Energy } \\
\text { Function }(\mathrm{n}=1000)\end{array}$ & $22(0.3407)$ & $6.5946 \mathrm{E}-07$ & $4(34.4602)$ & 3.1098E-09 & $6(1.5520)$ & $3.6708 \mathrm{E}-06$ \\
\hline $\begin{array}{l}\text { 7. Powell Function } \\
(\mathrm{n}=1000)\end{array}$ & $44(2.4865)$ & $6.2502 \mathrm{E}-07$ & $16(110.4723)$ & $3.8521 \mathrm{E}-07$ & $\begin{array}{c}25(3.2259) \\
\text { (failed) }\end{array}$ & $1.7934 \mathrm{E}-04$ \\
\hline $\begin{array}{l}\text { 8. Quartic With Noise } \\
\text { Function }(\mathrm{n}=1000)\end{array}$ & $383(0.2384)$ & $3.1145 \mathrm{E}-06$ & $13(91.8643)$ & $5.4263 \mathrm{E}-07$ & $1(0.1120)$ & 0 \\
\hline $\begin{array}{l}\text { 9. Rastrigin Function } \\
(\mathrm{n}=1000)\end{array}$ & $9(0.0712)$ & 3.5796E-08 & $1(21.1994)$ & 3.5728E-09 & $2(0.1493)$ & 0 \\
\hline $\begin{array}{l}\text { 10.Rotated Hyper Ellipsoid } \\
\text { Function }(\mathrm{n}=1000)\end{array}$ & $28(1.9075)$ & $4.3487 \mathrm{E}-08$ & $2(176.9553)$ & 4.3032E-13 & $\begin{array}{l}99 \begin{array}{l}(25.9046) \\
\text { (failed) }\end{array}\end{array}$ & 0.2211 \\
\hline $\begin{array}{l}\text { 11.Schwefel Function } \\
(\mathrm{n}=1000)\end{array}$ & $36(0.3373)$ & $9.4745 \mathrm{E}-07$ & $5(40.8946)$ & $9.0242 \mathrm{E}-07$ & $5(0.5322)$ & 0 \\
\hline $\begin{array}{l}\text { 12.Sphere Function } \\
(\mathrm{n}=1000)\end{array}$ & $14(0.0230)$ & 8.4047E-07 & $4(33.5744)$ & $2.9759 \mathrm{E}-08$ & $2(0.1065)$ & $1.4901 \mathrm{E}-08$ \\
\hline $\begin{array}{l}\text { 13. Styblinski Tang } \\
\text { Function }(\mathrm{n}=1000)\end{array}$ & $68(1.6197)$ & $2.6205 \mathrm{E}-06$ & $8(65.4069)$ & $1.0887 \mathrm{E}-06$ & $\begin{array}{c}\text { (2.5245) } \\
\text { (failed) }\end{array}$ & $1.7776 \mathrm{E}-04$ \\
\hline $\begin{array}{c}\text { 14. Sum Squares } \\
\text { Function }(\mathrm{n}=1000)\end{array}$ & $22(0.0300)$ & 7.9787E-07 & $4(35.9020)$ & $1.0839 \mathrm{E}-20$ & $\begin{array}{c}99(4.3612) \\
\text { (failed) }\end{array}$ & 0.4370 \\
\hline $\begin{array}{l}\text { 15. Schubert Function } \\
(\mathrm{n}=1000)\end{array}$ & $16(0.2256)$ & $8.2217 \mathrm{E}-07$ & $5(41.7283)$ & $2.1316 \mathrm{E}-14$ & $6(1.9671)$ & $5.8561 \mathrm{E}-05$ \\
\hline $\begin{array}{l}\text { 16. Stretched V Function } \\
\quad(\mathrm{n}=1000)\end{array}$ & $14(0.6951)$ & 4.2645E-07 & $3(73.4679)$ & 4.8573E-09 & $4(3.8702)$ & $2.0675 \mathrm{E}-07$ \\
\hline
\end{tabular}

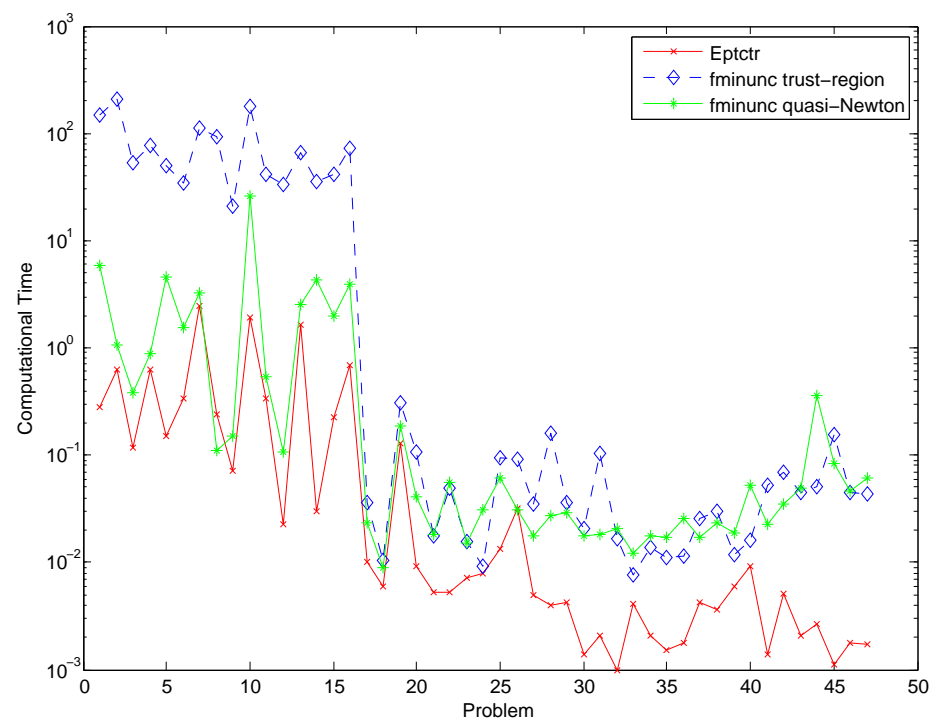

Fig. 1: The computational time of each problem. 
Table 2: Numerical results of small-scale problems with $n \leq 10$.

\begin{tabular}{|c|c|c|c|c|c|c|}
\hline \multirow{2}{*}{ Problems } & \multicolumn{2}{|c|}{ Eptctr } & \multicolumn{2}{|c|}{ fminunc.m (trust-region) } & \multicolumn{2}{|c|}{ fminunc.m (quasi-Newton) } \\
\hline & Iter (time (s)) & $\mid g\left(x^{i t}\right) \|_{\infty}$ & Iter (time (s)) & $\left\|g\left(x^{i t}\right)\right\|_{\infty}$ & Iter (time) & $\left\|g\left(x^{i t}\right)\right\|_{\infty}$ \\
\hline $\begin{array}{l}\text { 17. Beale Function } \\
(\mathrm{n}=2)\end{array}$ & $94(0.0100)$ & $3.4136 \mathrm{E}-08$ & $17(0.0359)$ & 2.3924E-05 & $21(0.0232)$ & $7.1145 \mathrm{E}-05$ \\
\hline $\begin{array}{l}\text { 18. Booth Function } \\
(\mathrm{n}=2)\end{array}$ & $24(0.0060)$ & 4.1473E-07 & $1(0.0105)$ & 0 & $1(0.0090)$ & $1.2666 \mathrm{E}-06$ \\
\hline $\begin{array}{l}\text { 19. Branin Function } \\
(\mathrm{n}=2)\end{array}$ & $32(0.1275)$ & $1.8526 \mathrm{E}-07$ & $4(0.3062)$ & $9.9309 \mathrm{E}-08$ & $7(0.1863)$ & $4.0602 \mathrm{E}-06$ \\
\hline $\begin{array}{l}\text { 20. Easom Function } \\
\quad(\mathrm{n}=2)\end{array}$ & $27(0.0092)$ & 9.9323E-07 & $6(0.1055)$ & 2.6839E-09 & $3(0.0405)$ & 4.7432E-09 \\
\hline $\begin{array}{l}\text { 21. Griewank Function } \\
\qquad(\mathrm{n}=10)\end{array}$ & $28(0.0053)$ & $8.1615 \mathrm{E}-07$ & $5(0.0176)$ & $7.2012 \mathrm{E}-10$ & $13(0.0180)$ & $2.9802 \mathrm{E}-07$ \\
\hline $\begin{array}{l}\text { 22. Hosaki Function } \\
(\mathrm{n}=2)\end{array}$ & $25(0.0053)$ & $8.2774 \mathrm{E}-07$ & $1(0.0489)$ & 8.5432E-09 & $5(0.0552)$ & $3.7253 \mathrm{E}-07$ \\
\hline $\begin{array}{l}\text { 23. Levy13 Function } \\
\qquad(\mathrm{n}=2)\end{array}$ & $22(0.0071)$ & $8.9312 \mathrm{E}-07$ & $3(0.0158)$ & $1.4647 \mathrm{E}-11$ & $2(0.0153)$ & $7.8931 \mathrm{E}-07$ \\
\hline $\begin{array}{l}\text { 24. Matyas Function } \\
(\mathrm{n}=2)\end{array}$ & $48(0.0080)$ & $7.5460 \mathrm{E}-07$ & $1(0.0092)$ & $4.7684 \mathrm{E}-08$ & $2(0.0310)$ & $2.1160 \mathrm{E}-07$ \\
\hline $\begin{array}{l}\text { 25. Mccormick Function } \\
(\mathrm{n}=2)\end{array}$ & $24(0.0132)$ & 4.2654E-07 & $\begin{array}{l}\text { (fo.0949) } \\
\text { (failed) }\end{array}$ & $6.0400 \mathrm{E}-04$ & $6(0.0617)$ & $1.8692 \mathrm{E}-08$ \\
\hline $\begin{array}{l}\text { 26. Perm Function } \\
(\mathrm{n}=4)\end{array}$ & $174(0.0320)$ & $2.5599 \mathrm{E}-08$ & $\begin{array}{c}20(0.0908) \\
\text { (failed) }\end{array}$ & 0.0112 & $\begin{array}{c}34(0.0307) \\
\text { (failed) }\end{array}$ & 0.0503 \\
\hline $\begin{array}{l}\text { 27. Power Sum Function } \\
\qquad(\mathrm{n}=4)\end{array}$ & $28(0.0050)$ & $2.0605 \mathrm{E}-07$ & $6(0.0353)$ & $2.2251 \mathrm{E}-07$ & $\begin{array}{l}\text { (0.0176) } \\
\text { (failed) }\end{array}$ & $1.2351 \mathrm{E}-04$ \\
\hline $\begin{array}{l}\text { 28. Price Function } \\
(\mathrm{n}=2)\end{array}$ & $61(0.0040)$ & 4.4811E-07 & $11(0.1592)$ & $7.8428 \mathrm{E}-06$ & $\begin{array}{c}27(0.0271) \\
\text { failed) }\end{array}$ & $9.0677 \mathrm{E}-04$ \\
\hline $\begin{array}{l}\text { 29. Zakharov Function } \\
(\mathrm{n}=10)\end{array}$ & $48(0.0042)$ & $2.7717 \mathrm{E}-07$ & $15(0.0363)$ & $5.9171 \mathrm{E}-06$ & $\begin{array}{c}26(0.0291) \\
\text { (failed) }\end{array}$ & 3.3111 \\
\hline $\begin{array}{l}\text { 30. Bohachevsky Function } \\
\qquad(\mathrm{n}=2)\end{array}$ & $32(0.0014)$ & 7.8927E-07 & $9(0.0204)$ & 2.1823E-06 & $8(0.0174)$ & $9.9838 \mathrm{E}-07$ \\
\hline $\begin{array}{l}\text { 31. Colville Function } \\
\quad(\mathrm{n}=4)\end{array}$ & $37(0.0021)$ & 8.0037E-08 & $\begin{array}{l}70(0.1032) \\
\text { (failed) }\end{array}$ & 0.0047 & $\begin{array}{l}27(0.0185) \\
\text { (failed) }\end{array}$ & 0.0014 \\
\hline $\begin{array}{l}\text { 32. Drop Wave } \\
\text { Function }(\mathrm{n}=2)\end{array}$ & $12(0.0010)$ & $3.6227 \mathrm{E}-07$ & $7(0.0168)$ & $2.7057 \mathrm{E}-08$ & $4(0.0206)$ & $1.2368 \mathrm{E}-06$ \\
\hline $\begin{array}{l}\text { 33. Schaffer Function } \\
\qquad(\mathrm{n}=2)\end{array}$ & $13(0.0041)$ & $3.3965 \mathrm{E}-07$ & $3(0.0076)$ & $4.0569 \mathrm{E}-10$ & $4(0.0120)$ & 3.3573E-09 \\
\hline $\begin{array}{l}\text { 34. Six Hump Camel } \\
\text { Function }(\mathrm{n}=2)\end{array}$ & $32(0.0021)$ & $1.7971 \mathrm{E}-07$ & $7(0.0139)$ & $2.6754 \mathrm{E}-06$ & $13(0.0176)$ & $7.5437 \mathrm{E}-05$ \\
\hline $\begin{array}{l}\text { 35. Three Hump Camel } \\
\text { Function }(\mathrm{n}=2)\end{array}$ & $28(0.0015)$ & $2.9007 \mathrm{E}-07$ & $5(0.0110)$ & $1.0843 \mathrm{E}-10$ & $10(0.0172)$ & $5.8845 \mathrm{E}-06$ \\
\hline $\begin{array}{l}\text { 36. Trecanni Function } \\
(\mathrm{n}=2)\end{array}$ & $27(0.0018)$ & $5.9765 \mathrm{E}-07$ & $7(0.0116)$ & $1.2378 \mathrm{E}-10$ & $18(0.0259)$ & $2.1014 \mathrm{E}-06$ \\
\hline $\begin{array}{l}\text { 37. Box Betts Exponential } \\
\text { Quadratic Sum Function }(\mathrm{n}=3)\end{array}$ & $12(0.0042)$ & $8.5907 \mathrm{E}-08$ & $7(0.0255)$ & $8.7407 \mathrm{E}-12$ & $14(0.0173)$ & $1.4076 \mathrm{E}-07$ \\
\hline $\begin{array}{l}\text { 38. Chichinadz Function } \\
(\mathrm{n}=2)\end{array}$ & $33(0.0036)$ & $2.1310 \mathrm{E}-07$ & $12(0.0300)$ & $1.4076 \mathrm{E}-13$ & $11(0.0236)$ & $1.2013 \mathrm{E}-05$ \\
\hline $\begin{array}{l}\text { 39. Eggholder Function } \\
(\mathrm{n}=2)\end{array}$ & $78(0.0059)$ & $1.3660 \mathrm{E}-05$ & $4(0.0118)$ & $8.3427 \mathrm{E}-07$ & $7(0.0188)$ & $6.0934 \mathrm{E}-08$ \\
\hline $\begin{array}{l}\text { 40. Exp2 Function } \\
(\mathrm{n}=2)\end{array}$ & $172(0.0093)$ & $2.0727 \mathrm{E}-07$ & $7(0.0160)$ & $3.4218 \mathrm{E}-09$ & $14(0.0530)$ & $1.1368 \mathrm{E}-07$ \\
\hline $\begin{array}{l}\text { 41. Hansen Function } \\
\qquad(\mathrm{n}=2)\end{array}$ & $15(0.0014)$ & $7.1112 \mathrm{E}-07$ & $7(0.0517)$ & $2.2678 \mathrm{E}-05$ & $8(0.0229)$ & $1.1921 \mathrm{E}-06$ \\
\hline $\begin{array}{l}\text { 42. Hartmann 3-D } \\
\text { dimensional Function }(\mathrm{n}=3)\end{array}$ & $54(0.0051)$ & $6.1888 \mathrm{E}-05$ & $12(0.0698)$ & $1.6596 \mathrm{E}-05$ & $15(0.0345)$ & $9.2387 \mathrm{E}-07$ \\
\hline $\begin{array}{l}\text { 43. Holder Table } \\
\text { Function }(\mathrm{n}=2)\end{array}$ & $16(0.0021)$ & $3.6270 \mathrm{E}-07$ & $8(0.0450)$ & 2.6754E-09 & $\begin{array}{c}\text { (0.3598) } \\
\text { (failed) }\end{array}$ & $7.4925 \mathrm{E}+37$ \\
\hline $\begin{array}{l}\text { 44. Michalewicz Function } \\
\qquad(\mathrm{n}=2)\end{array}$ & $31(0.0027)$ & $4.7446 \mathrm{E}-07$ & $10(0.0513)$ & $1.5065 \mathrm{E}-08$ & $12(0.0498)$ & $2.7057 \mathrm{E}-08$ \\
\hline $\begin{array}{l}\text { 45. Schaffer Function N.4 } \\
\qquad(\mathrm{n}=2)\end{array}$ & $2(0.0011)$ & $1.0267 \mathrm{E}-07$ & $46(0.1563)$ & $9.8423 \mathrm{E}-07$ & $10(0.0825)$ & $6.0786 \mathrm{E}-05$ \\
\hline $\begin{array}{l}\text { 46. Trefethen 4 Function } \\
\qquad(\mathrm{n}=2)\end{array}$ & $29(0.0018)$ & $2.4417 \mathrm{E}-07$ & $\begin{array}{c}8(0.0443) \\
\text { (failed) }\end{array}$ & $1.2562 \mathrm{E}-04$ & $\begin{array}{c}14(0.0464) \\
\text { (failed) }\end{array}$ & 0.0052 \\
\hline $\begin{array}{l}\text { 47. Zettl Function } \\
(\mathrm{n}=2)\end{array}$ & $40(0.0017)$ & $5.1869 \mathrm{E}-07$ & $13(0.0438)$ & $3.7740 \mathrm{E}-08$ & $18(0.0608)$ & $9.7767 \mathrm{E}-06$ \\
\hline
\end{tabular}


However, the trust-region method needs to solve a trust-region subproblem, which requires to solve a nonlinear system of equations at every iteration. The line search method involves one matrix-vector product to obtain the search direction $d_{k}$ and it involves about $n^{2}$ flops at every iteration. Furthermore, the step size $\alpha_{k}$ of the line search method is tried from 1 and reduced by the half with many times at every iteration. Since the linear model $f\left(x_{k}\right)+g_{k}^{T} s_{k}$ may not approximate $f\left(x_{k}+s_{k}\right)$ well in the transient-state phase, the step size $\alpha_{k}$ will be small. Consequently, the line search strategy consumes the unnecessary trial steps in the transient-state phase.

Numerical results also show that Eptctr is more robust and faster than the traditional optimization method such as the trust-region method and the line search method. The computational time of Eptctr is about one percent of that of the trustregion method (fminunc.m is set by the trust-region method ) or one fifth of that of the line search method (fminunc.m is set by the quasi-Newton method) for the test problems. Therefore, Eptctr can be regarded as a work horse for the unconstrained optimization problem and it is worth exploring further. We will extend it to the constrained optimization problem in the future.

\section{Acknowledgments}

This work was supported in part by Grant 61876199 from National Natural Science Foundation of China, Grant YBWL2011085 from Huawei Technologies Co., Ltd., and Grant YJCB2011003HI from the Innovation Research Program of Huawei Technologies Co., Ltd.. The authors are grateful to the anonymous referees for their comments and suggestions which greatly improve the presentation of this paper.

\section{References}

1. E.P. Adorio and U.P. Diliman, $M V F$-multivariate test functions library in $C$ for unconstrained global optimization, http://www.geocities.ws/eadorio/mvf .pdf, 2005.

2. E.L. Allgower and K. Georg, Introduction to Numerical Continuation Methods, SIAM, Philadelphia, PA, 2003.

3. U.M. Ascher and L.R. Petzold, Computer Methods for Ordinary Differential Equations and Differential-Algebraic Equations, SIAM, Philadelphia, PA, 1998.

4. C.A. Botsaris, A class of methods for unconstrained minimization based on stable numerical integration techniques, J. Math. Anal. Appl. 63 (1978) 729-749.

5. F.H. Branin, Widely convergent method for finding multiple solutions of simultaneous nonlinear equations, IBM J. Res. Dev. 16 (1972) 504-521.

6. C.G. Broyden, The convergence of a class of double-rank minimization algorithms, J. Inst. Math. Appl. 6 (1970) 76-90.

7. A.A. Brown and M.C. Bartholomew-Biggs, Some effective methods for unconstrained optimization based on the solution of systems of ordinary differential equations, J. Optim. Theory Appl. 62 (1989) 211-224.

8. J.C. Butcher and Z. Jackiewicz, Construction of high order diagonally implicit multistage integration methods for ordinary differential equations, Appl. Numer. Math. 27 (1998) 1-12.

9. R. Byrd, J. Nocedal and Y.X. Yuan, Global convergence of a class of quasi-Newton methods on convex problems, SIAM J. Numer. Anal. 24 (1987) 1171-1189.

10. T.F. Coleman and Y. Li. On the convergence of reflective Newton methods for large-scale nonlinear minimization subject to bounds, Math. Program. 67 (1994) 189-224. 
11. T.F. Coleman and Y. Li. An interior, trust region approach for nonlinear minimization subject to bounds, SIAM J. Optim. 6 (1996) 418-445.

12. A.R. Conn, N. Gould and Ph.L. Toint, Trust-Region Methods, SIAM, Philadelphia, USA, 2000.

13. W.C. Davidon, Variable metric method for minimization, SIAM J. Optim. 1 (1991) 1-17.

14. D.F. Davidenko, On a new method of numerical solution of systems of nonlinear equations (in Russian), Dokl. Akad. Nauk SSSR 88 (1953) 601-602.

15. R. Fletcher, A new approach to variable metric algorithms, Comput. J. 13 (1970) 317-322.

16. R. Fletcher and M.J.D. Powell, A rapidly convergent descent method for minimization, Comput. J. 6 (1963) 163-168.

17. B.S. Goh, Convergence of algorithms in optimizaton and solutions of nonlinear equations, J. Optim. Theory Appl. 144 (2010) 43-55.

18. D. Goldfarb, A family of variable metric updates derived by variational means, Math. Comput. 24 (1970) 23-26.

19. G.H. Golub and C.F. Van Loan, Matrix Computations, 4th ed., The Johns Hopkins University Press, 2013.

20. U. Helmke and J.B. Moore, Optimization and Dynamical Systems, 2nd ed., Springer-Verlag, London, 1996.

21. Z. Jackiewicz and S. Tracogna, A general class of two-step Runge-Kutta methods for ordinary differential equations, SIAM J. Numer. Anal. 32 (1995) 1390-1427.

22. D.J. Higham, Trust region algorithms and timestep selection, SIAM J. Numer. Anal. 37 (1999) 194 210

23. C. T. Kelley and D. E. Keyes, Convergence analysis of pseudo-transient continuation, SIAM J. Numer. Anal. 35 (1998) 508-523.

24. L.-Z. Liao, H.D. Qi, and L.Q. Qi, Neurodynamical optimization, J. Glob. Optim. 28 (2004) 175-195.

25. X.-L. Luo, L.-Z. Liao and H.-W. Tam, Convergence analysis of Levenberg-Marquardt methods, Optim. methods Softw. 22 (2007) 659-678.

26. X.-L. Luo, C.T. Kelley, L.-Z. Liao and H.-W. Tam, Combining trust-region techniques and Rosenbrock methods to compute stationary points, J. Optim. Theory Appl. 140 (2009) 265-286.

27. X.-L. Luo, J.H. Lv and G. Sun, Continuation methods with the trusty time-stepping scheme for linearly constrained optimization with noisy data, online published, Optim. Eng. (2021), available at http: //doi.org/10.1007/s11081-020-09590-z.

28. X.-L. Luo, H. Xiao and J.H. Lv, Continuation Newton methods with the residual trust-region timestepping scheme for nonlinear equations, June 2020, arXiv preprint, http://arxiv.org/abs/ 2006.02634.

29. X.-L. Luo and Y.Y. Yao, Primal-dual path-following methods and the trust-region updating strategy for linear programming with noisy data, available at http://arxiv .org/abs/2006.07568, Accepted, J. Comput. Math. (2021).

30. MATLAB 9.6.0 (R2019a), The MathWorks Inc., http: //www . mathworks . com, 2019.

31. N. Maculan and C. Lavor, A function to test methods applied to global minimization of potential energy of molecules, Numer. Algorithms 35 (2004) 287-300.

32. M.F. Mascarenhas, The BFGS method with exact line searches fails for non-convex objective functions, Math. Program. 99 (2004) 49-61.

33. J. Nocedal and S.J. Wright, Numerical Optimization, Springer-Verlag, 1999.

34. M.J.D. Powell, Convergence properties of a class of minimization algorithms, in: O.L. Mangasarian, R. R. Meyer and S. M. Robinson, eds., Nonlinear Programming 2, Academic Press, New York, 1-27, 1975.

35. J. Schropp, Using dynamical systems methods to solving minimization problems, Appl. Numer. Math. 18 (1995) 321-335.

36. D.F. Shanno, Conditioning of quasi-Newton methods for function minimization, Math. Comput. 24 (1970) 647-656.

37. S. Surjanovic and D. Bingham, Virtual library of simulation experiments: test functions and datasets, http://www.sfu.ca/ ssurjano, 2013.

38. L.F. Shampine, I. Gladwell and S. Thompson, Solving ODEs with MATLAB, Cambridge University Press, Cambridge, 2003.

39. W.Y. Sun and Y.X. Yuan, Optimization Theory and Methods: Nonlinear Programming, Springer, New York, 2006

40. K. Tanabe, Continuous Newton-Raphson method for solving an underdetermined system of nonlinear equations, Nonlinear Anal. 3 (1979) 495-503. 
41. K. Tanabe, A geometric method in nonlinear programming, J. Optim. Theory Appl. 30 (1980) 181210

42. N. Ullah, J. Sabi and A. Shah, A derivative-free scaled memoryless BFGS method for solving a system of monotone nonlinear equations, Submitted to Numer. Linear Algebra Appl., October 2020.

43. H. Yamashita, A differential equation approach to nonlinear programming, Math. Program. 18 (1980) 155-168, https://doi .org/10.1007/BF01588311.

44. Y. Yuan, Recent advances in trust region algorithms, Math. Program. 151 (2015) 249-281. 